\title{
Full Synchronization Studied by a Set of Partitions Connected Together
}

\author{
Jianbao Zhang, ${ }^{1}$ Zhongjun $\mathrm{Ma}^{2}$ and Jinde $\mathrm{Cao}^{3,4}$ \\ ${ }^{1}$ School of Science, Hangzhou Dianzi University, Hangzhou 310018, China \\ ${ }^{2}$ School of Mathematics and Computing Science, Guilin University of Electronic Technology, Guilin 541004, China \\ ${ }^{3}$ Department of Mathematics, Southeast University, Nanjing 210096, China \\ ${ }^{4}$ Department of Mathematics, King Abdulaziz University, Jeddah 21589, Saudi Arabia
}

Correspondence should be addressed to Zhongjun Ma; mzj1234402@163.com

Received 9 July 2013; Accepted 22 August 2013

Academic Editor: Qiankun Song

Copyright (C) 2013 Jianbao Zhang et al. This is an open access article distributed under the Creative Commons Attribution License, which permits unrestricted use, distribution, and reproduction in any medium, provided the original work is properly cited.

\begin{abstract}
A novel approach is brought forward for synchronization of a clustered network in this paper, the objective of which is twofold. The first one is to study cluster synchronization by analyzing the inner coupling matrices of the individual clusters instead of the one of the whole network. The other is to show that full synchronization can be ensured by several types of cluster synchronization, the partitions of which are connected together. Compared with the classical method for full synchronization, our approach reduces the network size to the cluster size and additionally obtains the thresholds for different types of cluster synchronization. As a numerical example, cluster and full synchronization in a special clustered network are investigated through our approach. It turns out that we obtain the same threshold for full synchronization as the one obtained by the classical method. Numerical simulations confirm the validity of our approach.
\end{abstract}

\section{Introduction}

Collective behavior of complex networks has become a focal subject due to the important and extensive applications in various fields of science and technology. Full and cluster synchronization are two types of typical and fundamental collective behavior. The former means that all oscillators in a network acquire identical behavior, while the latter means that the coupled oscillators split into subgroups called clusters, and all the oscillators in the same cluster behave in the same fashion. Research on cluster and full synchronization has attracted increasing attention in the past decades.

Several effective methods have been applied to study full synchronization, which is also called complete synchronization. Pecora and Carroll proposed the famous master stability function method to study the local stability of the synchronous state [1], while Lyapunov function method was employed to study the global stability of the synchronous state [2-4]. With the help of the two classical methods mentioned above, a great deal of research has been carried out in recent years. By imposing constraints on the coupling strength, local stability of the synchronous states in arbitrarily coupled systems was ensured based on Gershörin disk theory [5]. By analyzing the characters of the network topology, connection graph stability method was put forward [2] and applied to study synchronization in a network with timedependent on-off coupling, which is also called blinking model [3]. By decomposing the space into a direct sum of the synchronization manifold and the transverse space, criteria were obtained for both local and global stability of the synchronous state [4].

Cluster structures can be found to exist widely in realworld networks such as circles of friends or colleagues in social networks [6], compartments in food webs [7], groups of web pages sharing identical topics in the World Wide Web [8], and functional modules such as proteins having the same function in biological networks [9]. In those clustered networks, cluster synchronization usually occurs firstly before full synchronization occurs. Therefore, research on cluster and full synchronization in clustered networks has very obvious practical significance. Synchronization in a clustered network composed of two BA scale-free subnetworks has 
been studied in [10]. Control schemes were proposed for synchronization between two clusters, which is also called outer synchronization [11]. With the help of the method in [4], a criterion for cluster synchronization was obtained in [12]. Based on the criterion, cluster synchronization bifurcations are analyzed in a globally coupled network with a parameter [13].

However, to the best of our knowledge, most of the previous research on cluster and full synchronization focused on the topology of the whole network, which may be very complex. In this paper, the complexity of a clustered network is simplified by partitioning the whole network into clusters under a certain hypothesis. It is proved that cluster synchronization can be ensured by suitable inner couplings of the clusters, and sufficient conditions, which are independent of the outer couplings between different clusters, are obtained theoretically. Based on this result, a novel method for full synchronization is derived. If there exist two or several partitions connected together along some arrangement of all the oscillators, which imply that the intersection of the cluster synchronization manifolds corresponding to those partitions is equal to the full synchronization manifold, then full synchronization occurs if cluster synchronization corresponding to every partition is ensured. The method declares that both cluster and full synchronization can be studied by the inner topologies of the individual clusters. Obviously, the network size reduction provides convenience for the studies on synchronization in clustered networks with great mounts of oscillators.

The rest of the paper is organized as follows. Section 2 is devoted to introducing some concerned concepts such as partitions and cluster synchronization manifolds. Sufficient conditions independent of the outer couplings between different clusters are obtained for cluster synchronization in Section 3. The tedious proof of this result is carried out in the Appendix. The concept of partitions connected together along some arrangement is proposed and employed to study full synchronization in Section 4. In order to confirm the validity of the theoretical results, numerical experiments are carried out in Section 5. The final section is devoted to a brief discussion of the obtained results.

\section{Preliminaries}

Consider a network composed of $m$ oscillators

$$
\dot{x}_{i}=f\left(x_{i}, t\right)+\varepsilon \sum_{j=1}^{m} a_{i j} \Gamma x_{j}, \quad i=1, \ldots, m
$$

where $x_{i}=\left(x_{i}^{1}, \ldots, x_{i}^{n}\right)^{\top}$ is the state variable of the $i$ th oscillator, $t \in[0,+\infty)$ is a continuous time, $f: R^{n} \times$ $[0,+\infty) \rightarrow R^{n}$ is a continuous map, $\varepsilon \geq 0$ is the coupling strength, $\Gamma=\operatorname{diag}\left(\gamma_{1}, \ldots, \gamma_{n}\right)$ is a nonnegative matrix, $A=$ $\left(a_{i j}\right)_{m \times m}$ is the coupling matrix with $a_{i j}=a_{j i} \geq 0$ for $i \neq j$, and $\sum_{j=1}^{m} a_{i j}=s$ for $i=1, \ldots, m$.

Suppose that the index set $\{1, \ldots, m\}$ of the $m$ oscillators is divided into $d$ nonempty subsets called clusters. Let $P=$ $\left\{P_{1}, \ldots, P_{d}\right\}$ be its partition; that is, $P_{i} \cap P_{j}=\phi$ for $i \neq j$ and $\bigcup_{i=1}^{d} P_{i}=\{1, \ldots, m\}$. The following denotations are introduced for every $l=1, \ldots, d$.

$\left(D_{1}\right)$ Denote the cardinal number of cluster $P_{l}$ by $p_{l}$, and denote the subscript of the cluster containing $i$ by $\hat{i}$; that is, $\hat{i}=l$ if $i \in P_{l}$.

$\left(D_{2}\right)$ Suppose that all oscillators in the cluster $P_{l}$ are arranged adjacently; that is, $P_{l}=\left\{\sigma_{l-1}+1, \ldots, \sigma_{l}\right\}$, where $\sigma_{0}=0, \sigma_{l}=p_{1}+\cdots+p_{l}$.

$\left(D_{3}\right)$ Suppose that $p_{l} \geq 2$ for $1 \leq l \leq \bar{d}$ and $p_{l}=1$ for $\bar{d}<l \leq d$. It can be seen that for any $\bar{d}<l \leq d$, synchronization of the oscillators corresponding to cluster $P_{l}$ always occurs for any $\varepsilon \geq 0$ since $P_{l}$ contains only one oscillator.

We will discuss sufficient conditions for the $p_{l}$ oscillators corresponding to $P_{l}$ to synchronize with each other, $l=$ $1, \ldots, d$. Before that, the following sets should be introduced for every $l=1, \ldots, d$.

$\left(M_{1}\right)$ The synchronization submanifold of the cluster $P_{l}$ is as follows:

$\mathbb{M}\left(P_{l}\right)=\left\{\left(x_{\sigma_{l-1}+1}^{\top}, \ldots, x_{\sigma_{l}}^{\top}\right)^{\top} \in R^{p_{l} n} \mid x_{\sigma_{l-1}+1}=\cdots=x_{\sigma_{l}}\right\}$.

$\left(M_{2}\right)$ The cluster synchronization manifold of the partition $P$ is as follows:

$\mathbb{M}(P)=\mathbb{M}\left(P_{1}\right) \times \mathbb{M}\left(P_{2}\right) \times \cdots \times \mathbb{M}\left(P_{d}\right) \subset R^{m n}$.

$\left(M_{3}\right)$ The transverse subspace for $\mathbb{M}\left(P_{l}\right)$ is as follows:

$$
\mathbb{L}\left(P_{l}\right)=\left\{\left(x_{\sigma_{l-1}+1}^{\top}, \ldots, x_{\sigma_{l}}^{\top}\right)^{\top} \in R^{p_{l} n} \mid \sum_{i=1}^{p_{l}} x_{\sigma_{l-1}+i}=0\right\} .
$$

$\left(M_{4}\right)$ The transverse space for $\mathbb{M}(P)$ is as follows:

$$
\mathbb{L}(P)=\mathbb{L}\left(P_{1}\right) \times \mathbb{L}\left(P_{2}\right) \times \cdots \times \mathbb{L}\left(P_{d}\right) \subset R^{m n} .
$$

In case $d=1$, the synchronization manifold $\mathbb{M}(P)$ is called a full synchronization manifold. For simplicity, we denote the full synchronization manifold by $\mathbb{M}$ and the corresponding transverse space by $\mathbb{L}$.

Definitions of cluster and full synchronization in the network (1) are listed as follows.

$\left(S_{1}\right)$ The cluster synchronization manifold $\mathbb{M}(P)$ is said to be globally attractive for the network (1), or cluster synchronization of the partition $P$ occurs, if, for any initial condition $\left(x_{1}^{\top}(0), \ldots, x_{m}^{\top}(0)\right)^{\top}$,

$$
\lim _{t \rightarrow+\infty} \frac{1}{m} \sum_{l=1}^{d} \sum_{i \in P_{l}}\left\|x_{i}-x_{\sigma_{l-1}+1}\right\|=0
$$

where $\|\cdot\|$ denotes 2 -norm of vectors.

$\left(S_{2}\right)$ In case of $d=1$, the full synchronization manifold $\mathbb{M}$ is said to be globally attractive for the network (1); that is, full synchronization occurs. 


\section{Result on Cluster Synchronization}

Before the results on cluster synchronization are carried out, two common hypotheses in previous related research should be introduced.

At first, a synchronization manifold is always supposed to be an invariant manifold in order to discuss its attractiveness. The following lemma gives a sufficient and necessary condition for a cluster synchronization manifold being an invariant manifold.

Lemma 1 (see [12]). Partition the coupling matrix A according to partition $P$ as follows:

$$
A=\left[\begin{array}{cccc}
A_{11} & A_{12} & \cdots & A_{1 d} \\
A_{21} & A_{22} & \cdots & A_{2 d} \\
\cdots & \cdots & \cdots & \cdots \\
A_{d 1} & A_{d 2} & \cdots & A_{d d}
\end{array}\right]
$$

where $A_{l k} \in R^{p_{l} \times p_{k}}$; the synchronization manifold $\mathbb{M}(P)$ is an invariant manifold of the network (1), if and only if every submatrix $A_{l k}$ has equal-row-sum $s_{l k}, l, k=1, \ldots, d$. $\left(H_{1}\right)$

According to Lemma 1, we carry out the first hypothesis

$\left(H_{1}\right)$ Every submatrix $A_{l k} \in R^{p_{l} \times p_{k}}$ in the partitioned matrix (7) has equal-row-sum $s_{l k}$; that is, for every $i \in P_{l}$, there holds $\sum_{j \in P_{k}} a_{i j}=s_{l k}, l, k=1, \ldots, d$. In addition, suppose that all the principal submatrices $A_{l l}, l=1, \ldots, d$, are irreducible.

In order to study the inner couplings of the cluster $P_{l}$, we put forward the following matrix:

$$
\bar{A}\left(P_{l}\right)=A_{l l}+\sum_{k=1, k \neq l}^{d} s_{l k} I_{p_{l}}
$$

where $I_{p_{l}} \in R^{p_{l} \times p_{l}}$ is an identity matrix. Then the elements of $\bar{A}\left(P_{l}\right)$ satisfy that $\sum_{j \in P_{l}} \bar{a}_{i j}=s$ for $i \in P_{l}$, and

$$
\bar{a}_{i j}= \begin{cases}a_{i j}, & i \neq j, \\ a_{i j}+\sum_{k=1, k \neq l}^{d} s_{l k}, & i=j,\end{cases}
$$

where $i, j \in P_{l}, l=1, \ldots, d$. We will study the dependence of cluster synchronization on the matrices $\bar{A}\left(P_{l}\right), l=1, \ldots, d$. Noticing the importance of the matrix $\bar{A}\left(P_{l}\right)$, we call it a principal quasi-submatrix of the cluster $P_{l}$ since $A_{l l}$ is a principal submatrix, $l=1, \ldots, d$.

The second crucial hypothesis is the individual oscillator dynamics satisfying $\operatorname{QUAD}\left(\Delta, Q, R^{n}\right)$ condition as follows [12].

$\left(\mathrm{H}_{2}\right)$ There exists a positive-definite diagonal matrix $Q=$ $\operatorname{diag}\left(q_{1}, \ldots, q_{n}\right)$, with a diagonal matrix $\Delta=$ $\operatorname{diag}\left(\delta_{1}, \ldots, \delta_{n}\right)$ satisfying $\delta_{j} \leq 0$ for $j \in\left\{j: \gamma_{j}=0\right\}$ and a constant $\epsilon>0$, such that

$$
\begin{gathered}
(u-v)^{\top} Q\{[f(u, t)-f(v, t)]-\Delta(u-v)\} \\
\leq-\epsilon(u-v)^{\top}(u-v)
\end{gathered}
$$

holds for any $u, v \in R^{n}$ and all $t \geq 0$.

Hypothesis $\left(H_{2}\right)$ means that the following two coupled oscillators:

$$
\begin{aligned}
& \dot{x}_{1}=f\left(x_{1}, t\right)+\frac{\Delta\left(x_{2}-x_{1}\right)}{2}, \\
& \dot{x}_{2}=f\left(x_{2}, t\right)+\frac{\Delta\left(x_{1}-x_{2}\right)}{2}
\end{aligned}
$$

can synchronize when the coupling $\Delta / 2$ is made sufficiently large. Many chaotic oscillators have been proved to satisfy the hypothesis, such as Chua circuits [14], standard Hopfield neural networks [4], and $x$-coupled $[15,16]$ or $y$-coupled Lorenz systems [17] in an absorbing domain $\mathscr{B}$. However, many other systems are not the case such as a lattice of $x$-coupled Rössler systems, in which the stability of synchronization regime is lost with the increasing of coupling [18].

Now, the preliminaries above, together with Lyapunov function method, bring us to the following theorem.

Theorem 2. Suppose that hypotheses $\left(H_{1}\right)$ and $\left(H_{2}\right)$ hold and that

$$
\varepsilon \lambda_{2}\left(P_{l}\right) \gamma_{j}+\delta_{j} \leq 0, \quad j=1, \ldots, n,
$$

where $\lambda_{2}\left(P_{l}\right)$ is the second-largest eigenvalue of $\bar{A}\left(P_{l}\right), l=$ $1, \ldots, \bar{d}$, and the synchronization manifold $\mathbb{M}(P)$ is globally attractive for the network (1).

For a rigorous proof of Theorem 2, the reader is referred to the Appendix.

As a special case, if all the row sums of $A$ are equal to zero, then $\bar{A}\left(P_{l}\right)$ also has zero row sums, and $\lambda_{2}\left(P_{l}\right)<0$, $l=1, \ldots, \bar{d}$. Therefore, condition (12) is equivalent to

$$
\varepsilon \geq \frac{\max _{j \in\left\{j: \gamma_{j}>0\right\}}\left\{0, \delta_{j} / \gamma_{j}\right\}}{\min _{1 \leq l \leq \bar{d}}\left|\lambda_{2}\left(P_{l}\right)\right|} .
$$

\section{Result on Full Synchronization}

Suppose that there are a set of partitions $P(k, \cdot)=$ $\left\{P(k, 1), \ldots, P\left(k, d_{k}\right)\right\}, k=1, \ldots, c$, satisfying hypothesis $\left(H_{1}\right)$. The following definition is put forward for the first time to study full synchronization.

Definition 3. Rearrange the numbers $1 \cdots m$ as $i_{1} \cdots i_{m}$. A set of partitions $P(k), k=1, \ldots, c$, are connected together along the arrangement $i_{1} \cdots i_{m}$, if, for every $i_{j}, j=1, \ldots, m-1$, there exists a subset $P\left(k_{j}, l_{j}\right)$ such that $\left\{i_{j}, i_{j+1}\right\} \subseteq P\left(k_{j}, l_{j}\right)$.

For example, the set $\{1,2,3,4\}$ has two partitions as follows:

$$
P(1)=\{1,2 ; 3,4\}, \quad P(2)=\{1,3 ; 2,4\} .
$$


Since

$$
\{1,2\} \subseteq P(1,1), \quad\{2,4\} \subseteq P(2,2), \quad\{4,3\} \subseteq P(1,2),
$$

$P(1)$ and $P(2)$ form a set of partitions connected together along the arrangement 1243.

From the definition above, we obtain the following lemma.

Lemma 4. A set of partitions $P(k), k=1, \ldots, c$, are connected together along some arrangement $i_{1} \cdots i_{m}$, if and only if the cluster synchronization manifolds of those partitions satisfy that

$$
\bigcap_{k=1}^{c} \mathbb{M}(P(k))=\mathbb{M}
$$

The proof of Lemma 4 is straightforward and will not be given here.

Now, we are in a position to carry out the following theorem on full synchronization of the network (1).

Theorem 5. There are a set of partitions $P(k), k=1, \ldots, c$, connected together along some arrangement $i_{1} \cdots i_{m}$ and satisfying hypothesis $\left(H_{1}\right)$. Then under hypothesis $\left(H_{2}\right)$, the full synchronization manifold $\mathbb{M}$ is globally attractive for the network (1) if

$$
\varepsilon \gamma_{j} \lambda_{2}(P(k, l))+\delta_{j} \leq 0, \quad j=1, \ldots, n,
$$

where $\lambda_{2}(P(k, l))$ is the second-largest eigenvalue of $\bar{A}(P(k, l))$, $k=1, \ldots, c, l=1, \ldots, \bar{d}_{k}$.

The proof of Theorem 5 can be completed by combining Theorem 2 and Lemma 4, and so it is omitted here.

As a special case, if all the row-sums of $A$ are equal to zero, condition (17) is equivalent to

$$
\varepsilon \geq \frac{\max _{j \in\left\{j: \gamma_{j}>0\right\}}\left\{0, \delta_{j} / \gamma_{j}\right\}}{\min _{1 \leq k \leq c, 1 \leq l \leq \bar{d}_{k}}\left|\lambda_{2}(P(k, l))\right|} .
$$

\section{Numerical Examples}

Consider the system (1) composed of $m$ neural networks

$$
\dot{x}_{i}=-D x_{i}+\operatorname{Tg}\left(x_{i}\right)+\varepsilon \sum_{j=1}^{m} a_{i j} \Gamma x_{j}, \quad i=1, \ldots, m,
$$

where $x_{i} \in R^{3}, D=\Gamma=I_{3}, g\left(x_{i}\right)=\left(g\left(x_{i}^{1}\right), g\left(x_{i}^{2}\right), g\left(x_{i}^{3}\right)\right)^{\top}$, $g(s)=(|s+1|-|s-1|) / 2$, and

$$
T=\left(\begin{array}{ccc}
1.25 & -3.2 & -3.2 \\
-3.2 & 1.1 & -4.4 \\
-3.2 & 4.4 & 1.0
\end{array}\right)
$$

With the help of matlab LMI Control Toolbox, hypothesis $\left(H_{2}\right)$ can be satisfied by taking $Q=I_{3}$ and $\Delta=\delta I_{3}$, where $\delta=5.5685[4]$.

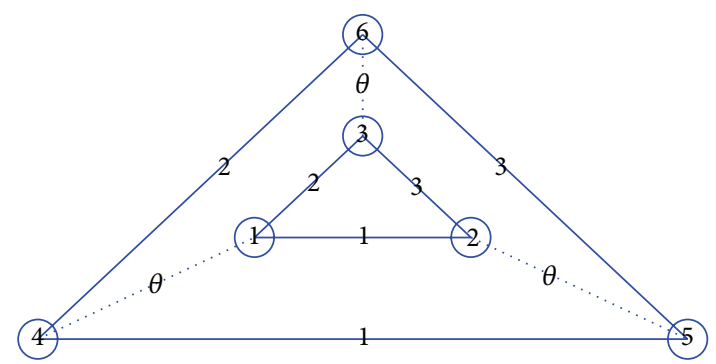

FIGURE 1: Topology structure corresponding to the coupling matrix (21). The coupling weights of the edges are the values lying on them.

Define the coupling matrix $A=\left(a_{i j}\right)_{6 \times 6}$ as follows:

$$
\begin{gathered}
A=\left(\begin{array}{ll}
A_{1} & \theta I_{3} \\
\theta I_{3} & A_{1}
\end{array}\right), \\
A_{1}=\left(\begin{array}{ccc}
-3-\theta & 1 & 2 \\
1 & -4-\theta & 3 \\
2 & 3 & -5-\theta
\end{array}\right) .
\end{gathered}
$$

The topology structure corresponding to the matrix (21) is shown in Figure 1. It is easy to show that the following partitions satisfy $\left(H_{1}\right)$ :

$$
P(1)=\{1,2,3 ; 4,5,6\}, \quad P(2)=\{1,4 ; 2,5 ; 3,6\},
$$

the principal quasi-submatrices of which are

$$
\begin{array}{cl}
\bar{A}\left(P\left(1, l_{1}\right)\right)=\left.A_{1}\right|_{\theta=0}, & l_{1}=1,2, \\
\bar{A}\left(P\left(2, l_{2}\right)\right)=\left(\begin{array}{cc}
-\theta & \theta \\
\theta & -\theta
\end{array}\right), & l_{2}=1,2,3,
\end{array}
$$

respectively. Further calculations give rise to the eigenvalues sets of the principal quasi-submatrices mentioned above as follows:

$$
\begin{gathered}
\sigma\left(\bar{A}\left(P\left(1, l_{1}\right)\right)\right)=\{0,-6 \pm \sqrt{3}\}, \quad l_{1}=1,2, \\
\sigma\left(\bar{A}\left(P\left(2, l_{2}\right)\right)\right)=\{0,-2 \theta\}, \quad l_{2}=1,2,3 .
\end{gathered}
$$

Obviously, partitions $P(1)$ and $P(2)$ are connected together along the arrangement 123654 . According to Theorems 2 and 5, one concludes the following.

(1) If $\varepsilon \geq \delta /(6-\sqrt{3})$, cluster synchronization of partition $P(1)$ occurs.

(2) If $\varepsilon \geq \delta /(2 \theta)$, cluster synchronization of partition $P(2)$ occurs.

(3) If $\varepsilon \geq \max \{\delta /(6-\sqrt{3}), \delta /(2 \theta)\}$, full synchronization occurs.

These results can be seen more clearly in Figure 2, the red solid lines in which are threshold lines of $\varepsilon-\theta$ for full synchronization.

In order to be compared with the previous classical results on full (complete) synchronization, the second-largest eigenvalue of $A$ should be obtained, and the threshold for full 


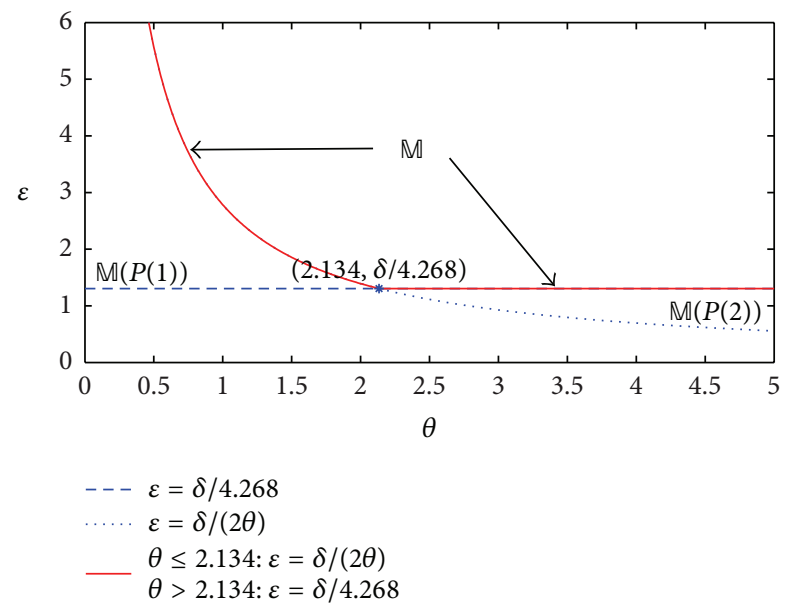

Figure 2: Thresholds for the attractiveness of the synchronization manifolds $\mathbb{M}(P(1)), \mathbb{M}(P(2))$, and $\mathbb{M}$.

synchronization should be $\varepsilon \geq \delta /\left|\lambda_{2}(A)\right|[1,4]$. In fact, the eigenvalues set of $A$ is

$$
\sigma(A)=\{0,-2 \theta,-6 \pm \sqrt{3},-6 \pm \sqrt{3}-2 \theta\} .
$$

Therefore, $\lambda_{2}(A)=\max \{-6+\sqrt{3},-2 \theta\}$. Obviously, there is a good agreement between our result and the previous classical results.

Define the following cluster errors:

$$
\begin{aligned}
& e_{1}\left(t_{0}\right)=\frac{1}{6} \sum_{i=1}^{3}\left\|x_{i}\left(t_{0}\right)-x_{1}\left(t_{0}\right)\right\|+\frac{1}{6} \sum_{i=4}^{6}\left\|x_{i}\left(t_{0}\right)-x_{4}\left(t_{0}\right)\right\|, \\
& e_{2}\left(t_{0}\right)=\frac{1}{6} \sum_{i=1}^{3}\left\|x_{i+3}\left(t_{0}\right)-x_{i}\left(t_{0}\right)\right\|, \\
& e\left(t_{0}\right)=\frac{1}{6} \sum_{i=1}^{6}\left\|x_{i}\left(t_{0}\right)-x_{1}\left(t_{0}\right)\right\|,
\end{aligned}
$$

where $t_{0}=100$. Take $\theta=0.1$ and let $\varepsilon$ increase from 0 to 1 step by step. Figure $3(\mathrm{a})$ shows that the cluster error $e_{1}\left(t_{0}\right)$ reaches zero firstly, while $e_{2}\left(t_{0}\right)$ and $e\left(t_{0}\right)$ reach zero at the same value of $\varepsilon$. While fixing $\theta$ at 3 , Figure $3(\mathrm{~b})$ shows that $e_{2}\left(t_{0}\right)$ reaches zero firstly, while $e_{1}\left(t_{0}\right)$ and $e\left(t_{0}\right)$ reach zero at the same value of $\varepsilon$.

In fact, these results can be forecasted in Figure 2. If the parameter $\theta$ is fixed in $(0,1.5]$ and if $\varepsilon$ increases gradually, synchronization of the partition $P(1)$ will firstly occur, and then synchronization of $P(2)$ and full synchronization occur at the same value of $\varepsilon$ since $\mathbb{M}(P(1)) \cap \mathbb{M}(P(2))=\mathbb{M}$. Therefore, the effectiveness of the theoretical results is confirmed.

\section{Conclusions}

This paper has investigated cluster and full synchronization in a clustered network. In order to study cluster synchronization, we propose the concept of principal quasi-submatrices corresponding to the individual clusters, which represent the inner couplings of the individual clusters. Theoretically, sufficient conditions independent of the outer couplings between different clusters are obtained for cluster synchronization. In order to study full synchronization, we propose the concept of partitions connected together along some arrangement. If all types of cluster synchronization of those partitions are ensured, it is proved that full synchronization occurs. The results are more advantageous than the classical results. Firstly, it allows us to divide a network composed of great amounts of oscillators into some smaller subnetworks. The network size reduction provides convenience to reduce the great amounts of calculations. Secondly, our approach can be applied to study cluster synchronization corresponding to any possible partitions. In summary, this paper has proposed a novel, convenient, and double purpose approach for both cluster and full synchronization in clustered networks.

\section{Appendix}

Denoting $\iota_{p_{l}}=(1, \ldots, 1)^{\top} \in R^{p_{l}}$, we define the following cluster errors for $l=1, \ldots, d$ :

$$
\begin{aligned}
& \left(E_{1}\right) \bar{x}_{l}=\left(1 / p_{l}\right) \sum_{i \in P_{l}} x_{i}, \delta x_{i}=x_{i}-\bar{x}_{\hat{i}}, i=1, \ldots, m ; \\
& \left(E_{2}\right) X_{l}=\left(x_{\sigma_{l-1}+1}^{\top}, \ldots, x_{\sigma_{l}}^{\top}\right)^{\top}, \bar{X}_{l}=\iota_{p_{l}} \otimes \bar{x}_{l}, \delta X_{l}=X_{l}-\bar{X}_{l} ; \\
& \left(E_{3}\right) x=\left(x_{1}^{\top}, \ldots, x_{m}^{\top}\right)^{\top}, \bar{x}=\left(\bar{x}_{\widehat{1}}^{\top}, \ldots, \bar{x}_{\widehat{m}}^{\top}\right)^{\top}, \delta x= \\
& \quad\left(\delta x_{1}^{\top}, \ldots, \delta x_{m}^{\top}\right)^{\top} .
\end{aligned}
$$

Proof. Denote $F\left(X_{l}, t\right)=\left(f\left(x_{\sigma_{l-1}+1}, t\right)^{\top}, \ldots, f\left(x_{\sigma_{l}}, t\right)^{\top}\right)^{\top}$ and rewrite the network (1) as follows:

$$
\frac{d X_{l}}{d t}=F\left(X_{l}, t\right)+\varepsilon \sum_{k=1}^{d}\left(A_{l k} \otimes \Gamma\right) X_{k}
$$

Therefore,

$$
\begin{aligned}
\frac{d \delta X_{l}}{d t} & =\frac{d\left(X_{l}-\bar{X}_{l}\right)}{d t}=\frac{d X_{l}}{d t}-\iota_{p_{l}} \otimes\left(\frac{1}{p_{l}} \sum_{i \in P_{l}} \frac{d x_{i}}{d t}\right) \\
& =F\left(X_{l}, t\right)+\varepsilon \sum_{k=1}^{d}\left(A_{l k} \otimes \Gamma\right) X_{k}-\iota_{p_{l}} \otimes\left(\frac{1}{p_{l}} \sum_{i \in P_{l}} \frac{d x_{i}}{d t}\right) \\
& =F\left(X_{l}, t\right)-F\left(\bar{X}_{l}, t\right)+\varepsilon \sum_{k=1}^{d}\left(A_{l k} \otimes \Gamma\right) \delta X_{k}+\rrbracket_{l},
\end{aligned}
$$

where

$$
\sqrt{l}_{l}=F\left(\bar{X}_{l}, t\right)+\varepsilon \sum_{k=1}^{d}\left(A_{l k} \otimes \Gamma\right) \bar{X}_{k}-\iota_{p_{l}} \otimes\left(\frac{1}{p_{l}} \sum_{i \in P_{l}} \frac{d x_{i}}{d t}\right) .
$$




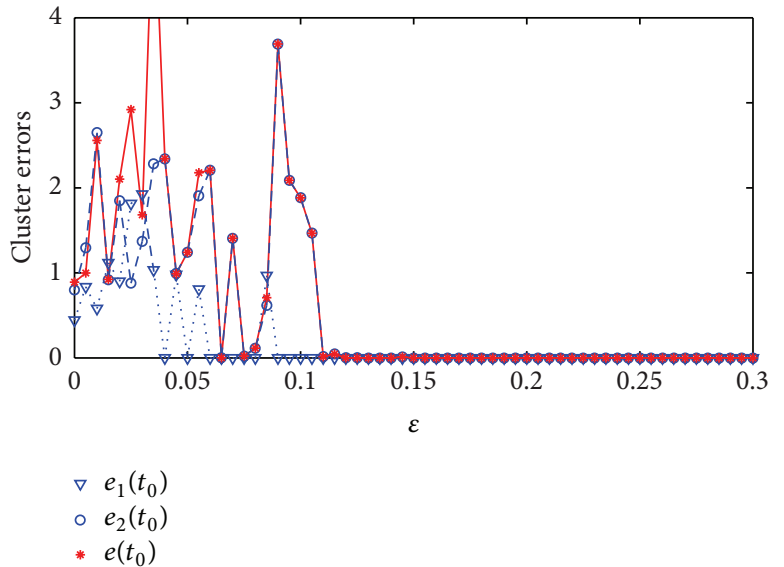

(a) $\theta=0.1$

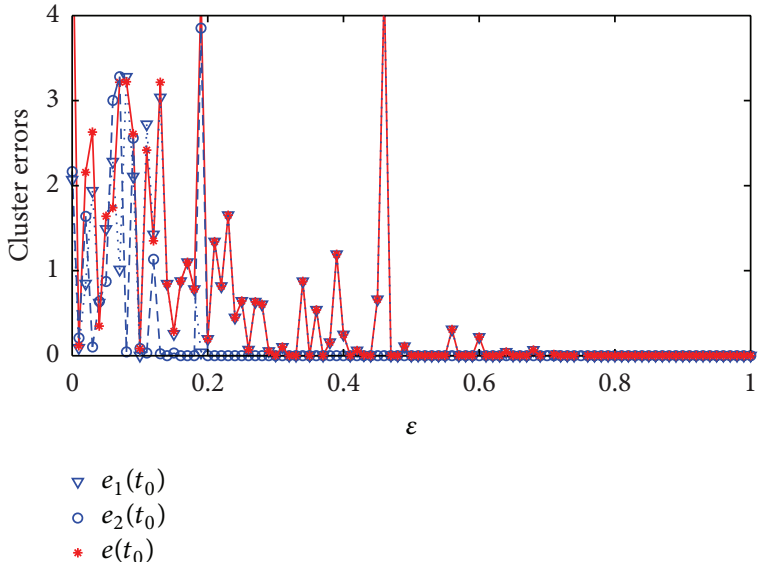

(b) $\theta=3$

FIGURE 3: Dependence of the cluster errors $e_{1}\left(t_{0}\right), e_{2}\left(t_{0}\right)$, and $e\left(t_{0}\right)$ on the coupling strength $\varepsilon$ for the network (19) with the coupling matrix (21).

Noticing that $F\left(\bar{X}_{l}, t\right)=\iota_{p_{l}} \otimes f\left(\bar{x}_{l}, t\right)$ and

$$
\begin{aligned}
\sum_{k=1}^{d}\left(A_{l k} \otimes \Gamma\right) \bar{X}_{k} \\
=\sum_{k=1}^{d}\left(A_{l k} \otimes \Gamma\right)\left(\iota_{p_{k}} \otimes \bar{x}_{k}\right)=\sum_{k=1}^{d}\left(A_{l k} \iota_{p_{k}}\right) \otimes\left(\Gamma \bar{x}_{k}\right) \\
=\sum_{k=1}^{d} s_{l k} \iota_{p_{l}} \otimes\left(\Gamma \bar{x}_{k}\right)=\iota_{p_{l}} \otimes\left(\sum_{k=1}^{d} s_{l k} \Gamma \bar{x}_{k}\right),
\end{aligned}
$$

we have

$$
\mathbb{J}_{l}=\iota_{p_{l}} \otimes\left(f\left(\bar{x}_{l}, t\right)+\sum_{k=1}^{d} s_{l k} \Gamma \bar{x}_{k}-\frac{1}{p_{l}} \sum_{i \in P_{l}} \frac{d x_{i}}{d t}\right) .
$$

Let $J_{l}=\iota_{p_{l}} \otimes J_{l}$; it can be seen that $J_{l}$ is independent of $i \in P_{l}$.

In order to utilize the $\operatorname{QUAD}\left(\Delta, Q, R^{n}\right)$ condition, a Lyapunov function is defined as follows,

$$
V(\delta x)=\frac{1}{2} \sum_{l=1}^{d} \delta X_{l}^{\top}\left(I_{p_{l}} \otimes Q\right) \delta X_{l} .
$$

The derivative of $V$ with respect to time $t$ is as follows:

$$
\begin{aligned}
\frac{d V(\delta x)}{d t}= & \sum_{l=1}^{d} \delta X_{l}^{\top}\left(I_{p_{l}} \otimes Q\right) \frac{d \delta X_{l}}{d t} \\
= & \sum_{l=1}^{d} \delta X_{l}^{\top}\left(I_{p_{l}} \otimes Q\right) \\
& \times\left(F\left(X_{l}, t\right)-F\left(\bar{X}_{l}, t\right)+\varepsilon \sum_{k=1}^{d}\left(A_{l k} \otimes \Gamma\right) \delta X_{k}+\rrbracket_{l}\right) .
\end{aligned}
$$

Noticing that $\sum_{i \in P_{l}} \delta x_{i}=0$, we have

$$
\begin{aligned}
& \sum_{l=1}^{d} \delta X_{l}^{\top}\left(I_{p_{l}} \otimes Q\right) \mathbb{J}_{l} \\
& =\sum_{l=1}^{d} \delta X_{l}^{\top}\left(I_{p_{l}} \otimes Q\right)\left(\iota_{p_{l}} \otimes J_{l}\right) \\
& =\sum_{l=1}^{d} \delta X_{l}^{\top}\left(\iota_{p_{l}} \otimes Q J_{l}\right)
\end{aligned}
$$

which together with hypothesis $\left(H_{2}\right)$ leads to

$$
\frac{d V(\delta x)}{d t}=\sum_{l=1}^{d} \delta X_{l}^{\top}\left(I_{p_{l}} \otimes Q\right)
$$

$$
\begin{gathered}
\times\left(\left[F\left(X_{l}, t\right)-F\left(\bar{X}_{l}, t\right)\right.\right. \\
\left.-\left(I_{p_{l}} \otimes \Delta\right)\left(X_{l}-\bar{X}_{l}\right)\right] \\
+\left[\left(I_{p_{l}} \otimes \Delta\right)\left(X_{l}-\bar{X}_{l}\right)\right. \\
\left.\left.+\varepsilon \sum_{k=1}^{d}\left(A_{l k} \otimes \Gamma\right) \delta X_{k}\right]\right)
\end{gathered}
$$




$$
\begin{aligned}
& \leq-\epsilon \sum_{l=1}^{d} \delta X_{l}^{\top} \delta X_{l}+\sum_{l=1}^{d} \delta X_{l}^{\top} \\
& \quad \times\left[\left(I_{p_{l}} \otimes Q \Delta\right) \delta X_{l}+\varepsilon \sum_{k=1}^{d}\left(A_{l k} \otimes Q \Gamma\right) \delta X_{k}\right] \\
& \leq-2 \epsilon \frac{V(\delta x(t))}{\max _{1 \leq i \leq n} q_{i}}+\mathbb{S} .
\end{aligned}
$$

Noticing the equality (8), that is, $A_{l l}=\bar{A}_{l}-\sum_{k=1, k \neq l}^{d} s_{l k} I_{p_{l}}$, where $\bar{A}_{l}=\bar{A}\left(P_{l}\right)$, we have

$$
\begin{aligned}
\mathbb{S}= & \sum_{l=1}^{d} \delta X_{l}^{\top}\left(\left(I_{p_{l}} \otimes Q \Delta\right) \delta X_{l}+\varepsilon\left(A_{l l} \otimes Q \Gamma\right) \delta X_{l}\right. \\
& \left.+\varepsilon \sum_{k=1, k \neq l}^{d}\left(A_{l k} \otimes Q \Gamma\right) \delta X_{k}\right) \\
= & \sum_{l=1}^{d} \delta X_{l}^{\top}\left[I_{p_{l}} \otimes Q \Delta+\varepsilon \bar{A}_{l} \otimes Q \Gamma\right] \delta X_{l} \\
& +\varepsilon \sum_{l=1}^{d} \sum_{k=1, k \neq l}^{d} \delta X_{l}^{\top}\left[\left(A_{l k} \otimes Q \Gamma\right) \delta X_{k}-s_{l k}\left(I_{p_{l}} \otimes Q \Gamma\right) \delta X_{l}\right] \\
= & \mathbb{S}_{1}+\mathbb{S}_{2} .
\end{aligned}
$$

The proof will be completed by showing that $\mathbb{S}_{1} \leq 0$ and $\mathbb{S}_{2} \leq$ 0 as follows.

As we know, the symmetric matrix $\bar{A}_{l}$ has the famous decomposition $\bar{A}_{l}=U_{l} \Lambda_{l} U_{l}^{\top}$, where $\Lambda_{l}=$ $\operatorname{diag}\left\{\lambda_{1}\left(P_{l}\right), \ldots, \lambda_{p_{l}}\left(P_{l}\right)\right\}$ satisfying $s=\lambda_{1}\left(P_{l}\right)>\lambda_{2}\left(P_{l}\right) \geq$ $\cdots \geq \lambda_{p_{l}}\left(P_{l}\right)$, and $U_{l} \in R^{p_{l} \times p_{l}}$ is a unitary matrix; that is, $U_{l} U_{l}^{\top}=I_{p}$. The $i$ th column of $U_{l}$ is the eigenvector of $\bar{A}_{l}$ corresponding to the eigenvalue $\lambda_{i}\left(P_{l}\right), i=1, \ldots, p_{l}$. By the substitutions of variables $\delta X_{l}=\left(U_{l} \otimes I_{n}\right) \xi_{l}, l=1, \ldots, d$, we have

$$
\begin{aligned}
\mathbb{S}_{1}= & \sum_{l=1}^{d} \xi_{l}^{\top}\left(U_{l} \otimes I_{n}\right)^{\top} \\
& \times\left[I_{p_{l}} \otimes(Q \Delta)+\varepsilon \bar{A}_{l} \otimes(Q \Gamma)\right]\left(U_{l} \otimes I_{n}\right) \xi_{l} \\
= & \sum_{l=1}^{d} \xi_{l}^{\top}\left[I_{p_{l}} \otimes(Q \Delta)+\varepsilon\left(U_{l}^{\top} \bar{A}_{l} U_{l}\right) \otimes(Q \Gamma)\right] \xi_{l} \\
= & \sum_{l=1}^{d} \xi_{l}^{\top}\left[I_{p_{l}} \otimes(Q \Delta)+\varepsilon \Lambda_{l} \otimes(Q \Gamma)\right] \xi_{l} .
\end{aligned}
$$

Noticing that the matrices $Q, \Delta, \Lambda_{l}$, and $\Gamma$ are all diagonal matrices, we obtain

$$
\mathbb{S}_{1}=\sum_{l=1}^{d} \xi_{l}^{\top} \operatorname{diag}(\underbrace{\lambda_{11}, \ldots, \lambda_{1 n}}_{n}, \ldots, \underbrace{\lambda_{p_{l} 1}, \ldots, \lambda_{p_{l} n}}_{n}) \xi_{l},
$$

where $\lambda_{i j}=q_{j}\left(\varepsilon \lambda_{i}\left(P_{l}\right) \gamma_{j}+\delta_{j}\right), i=1, \ldots p_{l}, j=1, \ldots, n$. It is well known that the first column of $U_{l}$ is $\iota_{p_{l}}$; then one can conclude from the inverse substitutions of variables $\xi_{l}=$ $\left(U_{l}^{\top} \otimes I_{n}\right) \delta X_{l}$ that $\xi_{l}^{j}=\sum_{i \in P_{l}} \delta x_{i}^{j}=0, j=1, \ldots, n, l=1, \ldots, d$. Therefore, condition (12) is sufficient for $\mathbb{S}_{1} \leq 0$.

Finally, some techniques in [2, page 164] are employed to show that $\mathbb{S}_{2} \leq 0$ as follows:

$$
\begin{aligned}
& \mathbb{S}_{2}=\varepsilon \sum_{l=1}^{d} \sum_{k=1, k \neq l}^{d} \delta X_{l}^{\top}\left[\left(A_{l k} \otimes Q \Gamma\right) \delta X_{k}-s_{l k}\left(I_{p_{l}} \otimes Q \Gamma\right) \delta X_{l}\right] \\
& =\varepsilon\left[\sum_{l=1}^{d-1} \sum_{k=l+1}^{d}+\sum_{k=1}^{d-1} \sum_{l=k+1}^{d}\right] \\
& \times \delta X_{l}^{\top}\left[\left(A_{l k} \otimes Q \Gamma\right) \delta X_{k}-s_{l k}\left(I_{p_{l}} \otimes Q \Gamma\right) \delta X_{l}\right] \\
& =\varepsilon \sum_{l=1}^{d-1} \sum_{k=l+1}^{d} \delta X_{l}^{\top}\left[\left(A_{l k} \otimes Q \Gamma\right) \delta X_{k}-s_{l k}\left(I_{p_{l}} \otimes Q \Gamma\right) \delta X_{l}\right] \\
& +\varepsilon \sum_{l=1}^{d-1} \sum_{k=l+1}^{d} \delta X_{k}^{\top}\left[\left(A_{k l} \otimes Q \Gamma\right) \delta X_{l}-s_{k l}\left(I_{p_{k}} \otimes Q \Gamma\right) \delta X_{k}\right] \\
& =\varepsilon \sum_{l=1}^{d-1} \sum_{k=l+1}^{d}\left(\delta X _ { l } ^ { \top } \left[\sum_{i \in P_{l}} \sum_{j \in P_{k}}\left(a_{i j} E_{i j}^{p_{l} \times p_{k}} \otimes Q \Gamma\right) \delta X_{k}\right.\right. \\
& \left.-s_{l k}\left(\sum_{i \in P_{l}} E_{i i}^{p_{1} \times p_{l}} \otimes Q \Gamma\right) \delta X_{l}\right] \\
& +\delta X_{k}^{\top}\left[\sum_{i \in P_{l}} \sum_{j \in P_{k}}\left(a_{j i} E_{j i}^{p_{k} \times p_{l}} \otimes Q \Gamma\right) \delta X_{l}\right. \\
& \left.\left.-s_{k l}\left(\sum_{j \in P_{k}} E_{j j}^{p_{k} \times p_{k}} \otimes Q \Gamma\right) \delta X_{k}\right]\right),
\end{aligned}
$$

where $E_{i j}^{p_{1} \times p_{k}}=e_{i}^{p_{l}} \otimes e_{j}^{p_{k} \top}$ and $e_{i}^{p} \in R^{p}$ is the $i$ th column of $I_{p}$. Substituting $s_{l k}$ and $s_{k l}$ by (9), we have

$$
\begin{array}{r}
\mathbb{S}_{2}=\varepsilon \sum_{l=1}^{d-1} \sum_{k=l+1}^{d} \sum_{i \in P_{l}} \sum_{j \in P_{k}} a_{i j}\left(\delta X _ { l } ^ { \top } \left[\left(E_{i j}^{p_{1} \times p_{k}} \otimes Q \Gamma\right) \delta X_{k}\right.\right. \\
\left.-\left(E_{i i}^{p_{l} \times p_{l}} \otimes Q \Gamma\right) \delta X_{l}\right] \\
+\delta X_{k}^{\top}\left[\left(E_{j i}^{p_{k} \times p_{l}} \otimes Q \Gamma\right) \delta X_{l}\right. \\
\left.\left.-\left(E_{j j}^{p_{k} \times p_{k}} \otimes Q \Gamma\right) \delta X_{k}\right]\right)
\end{array}
$$




$$
\begin{gathered}
=\varepsilon \sum_{l=1}^{d-1} \sum_{k=l+1}^{d} \sum_{i \in P_{l}} \sum_{j \in P_{k}} a_{i j}\left(\delta X_{l}^{\top}\left[e_{i}^{p_{l}} \otimes \mathrm{Q} \Gamma\left(\delta x_{j}-\delta x_{i}\right)\right]\right. \\
\left.+\delta X_{k}^{\top}\left[e_{j}^{p_{k}} \otimes \mathrm{Q} \Gamma\left(\delta x_{i}-\delta x_{j}\right)\right]\right) \\
=\varepsilon \sum_{l=1}^{d-1} \sum_{k=l+1}^{d} \sum_{i \in P_{l}} \sum_{j \in P_{k}} a_{i j}\left(\delta x_{i}^{\top} \mathrm{Q} \Gamma\left(\delta x_{j}-\delta x_{i}\right)\right. \\
\left.+\delta x_{j}^{\top} \mathrm{Q} \Gamma\left(\delta x_{i}-\delta x_{j}\right)\right) \\
=-\varepsilon \sum_{l=1}^{d-1} \sum_{k=l+1}^{d} \sum_{i \in P_{l}} \sum_{j \in P_{k}} a_{i j}\left(\delta x_{j}-\delta x_{i}\right)^{\top} \mathrm{Q} \Gamma\left(\delta x_{j}-\delta x_{i}\right) \leq 0 .
\end{gathered}
$$

The proof is completed.

\section{Acknowledgments}

This project was supported the by the National Natural Science Foundation of China (Grant nos. 11162004 and 60964006), Zhejiang Provincial Natural Science Foundation of China (no. LQ12a01003), the Science Foundation of Guangxi Province (no. 2013GXNSFAA019006), and the Deanship of Scientific Research (DSR), King Abdulaziz University, under Grant no. 3-130/1434/HiCi. The authors, therefore, acknowledge the technical and financial support of KAU.

\section{References}

[1] L. M. Pecora and T. L. Carroll, "Master stability functions for synchronized coupled systems," Physical Review Letters, vol. 80, no. 10, pp. 2109-2112, 1998.

[2] V. N. Belykh, I. V. Belykh, and M. Hasler, "Connection graph stability method for synchronized coupled chaotic systems," Physica D, vol. 195, no. 1-2, pp. 159-187, 2004.

[3] I. V. Belykh, V. N. Belykh, and M. Hasler, "Blinking model and synchronization in small-world networks with a time-varying coupling," Physica D, vol. 195, no. 1-2, pp. 188-206, 2004.

[4] W. Lu and T. Chen, "New approach to synchronization analysis of linearly coupled ordinary differential systems," Physica D, vol. 213, no. 2, pp. 214-230, 2006.

[5] Y. H. Chen, G. Rangarajan, and M. Z. Ding, "Stability analysis of synchronized dynamics in coupled systems," Physical Review E, vol. 67, Article ID 026209, 4 pages, 2003.

[6] M. Girvan and M. E. J. Newman, "Community structure in social and biological networks," Proceedings of the National Academy of Sciences of the United States of America, vol. 99, no. 12, pp. 7821-7826, 2002.

[7] A. E. Krause, K. A. Frank, D. M. Mason, R. E. Ulanowicz, and W. W. Taylor, "Compartments revealed in food-web structure," Nature, vol. 426, no. 6964, pp. 282-285, 2003.

[8] G. W. Flake, S. Lawrence, C. L. Giles, and F. M. Coetzee, "Selforganization of the web and identification of communities," IEEE Computer, vol. 35, no. 3, pp. 66-70, 2002.

[9] A. W. Rives and T. Galitski, "Modular organization of cellular networks," Proceedings of the National Academy of Sciences of the United States of America, vol. 100, no. 3, pp. 1128-1133, 2003.
[10] C. Feng, Y. L. Zou, and F. Q. Wei, "Synchronization processes in clustered networks with different inter-cluster couplings," Acta Physica Sinica, vol. 62, no. 7, Article ID 070506, 7 pages, 2013.

[11] X. Wu, W. X. Zheng, and J. Zhou, "Generalized outer synchronization between complex dynamical networks," Chaos, vol. 19, no. 1, article 013109, 9 pages, 2009.

[12] W. Wu and T. Chen, "Partial synchronization in linearly and symmetrically coupled ordinary differential systems," Physica D, vol. 238, no. 4, pp. 355-364, 2009.

[13] Z. J. Ma, S. Z. Zhang, and G. R. Jiang, "Effect of the coupling matrix with a weight parameter on synchronization pattern in a globally coupled network," Nonlinear Dynamics, 2013.

[14] C. W. Wu and L. O. Chua, "Synchronization in an array of linearly coupled dynamical systems," IEEE Transactions on Circuits and Systems. I, vol. 42, no. 8, pp. 430-447, 1995.

[15] I. Belykh, V. Belykh, K. Nevidin, and M. Hasler, "Persistent clusters in lattices of coupled nonidentical chaotic systems," Chaos, vol. 13, no. 1, pp. 165-178, 2003.

[16] Z. Ma, Z. Liu, and G. Zhang, "A new method to realize cluster synchronization in connected chaotic networks," Chaos, vol. 16, no. 2, aricle 023103, 9 pages, 2006.

[17] X. Liu and T. Chen, "Boundedness and synchronization of $y$ coupled Lorenz systems with or without controllers," Physica D, vol. 237, no. 5, pp. 630-639, 2008.

[18] J. F. Heagy, L. M. Pecora, and T. L. Carroll, "Short wavelength bifurcations and size instabilities in coupled oscillator systems," Physical Review Letters, vol. 74, no. 21, pp. 4185-4188, 1995. 


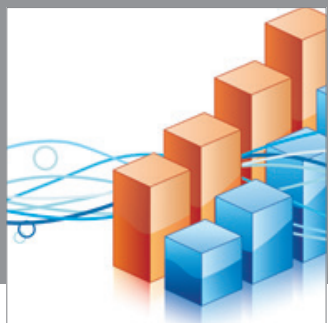

Advances in

Operations Research

mansans

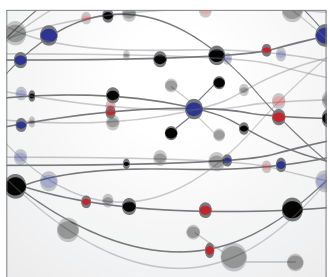

The Scientific World Journal
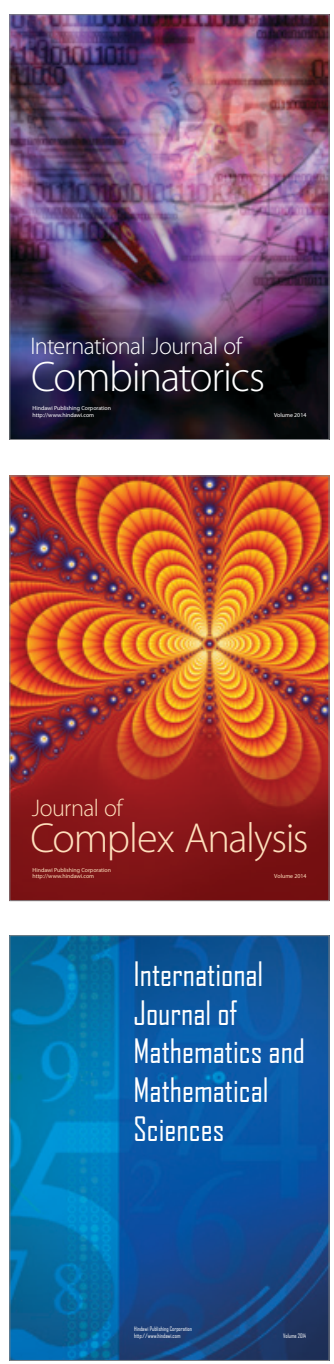
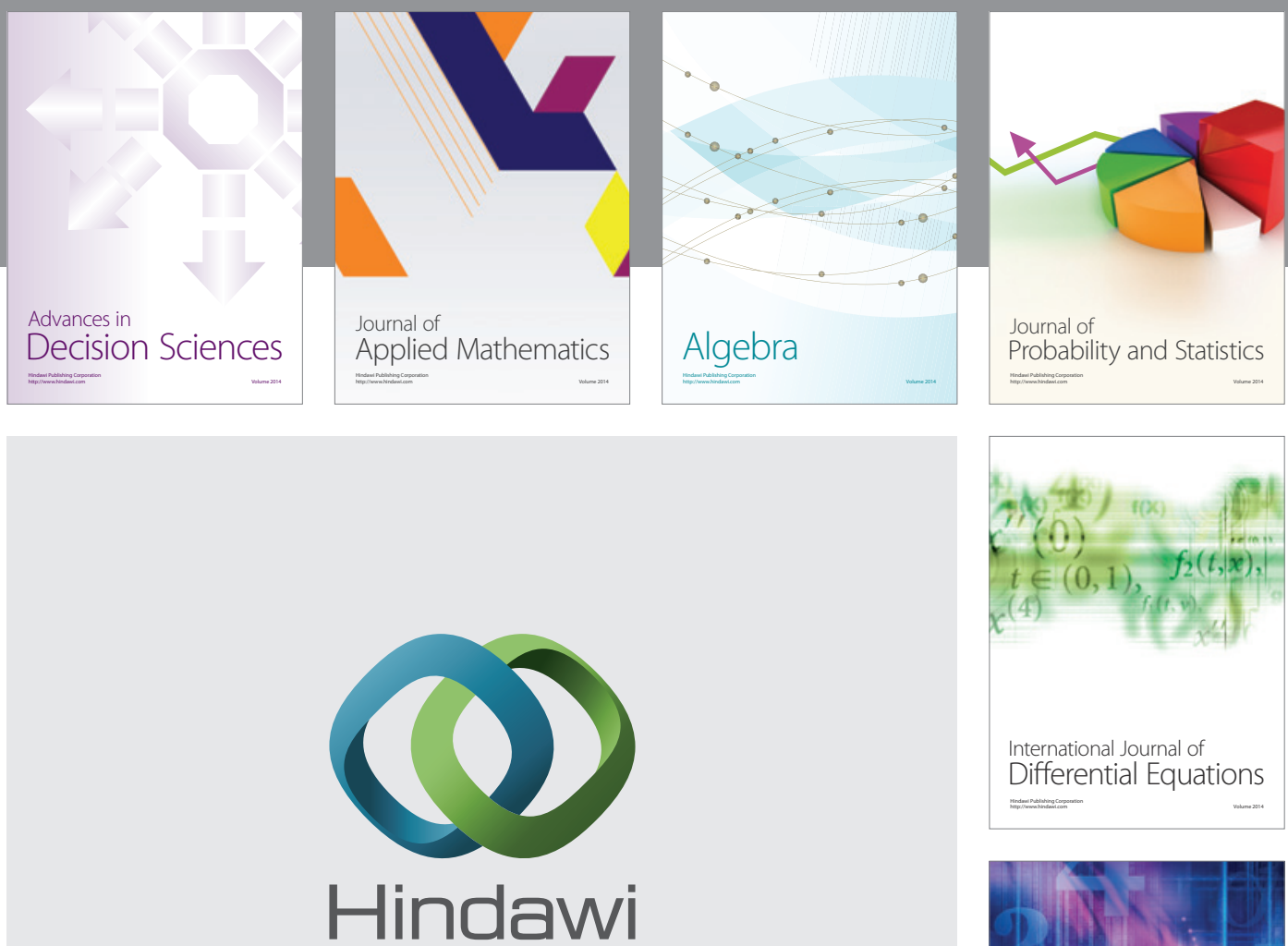

Submit your manuscripts at http://www.hindawi.com
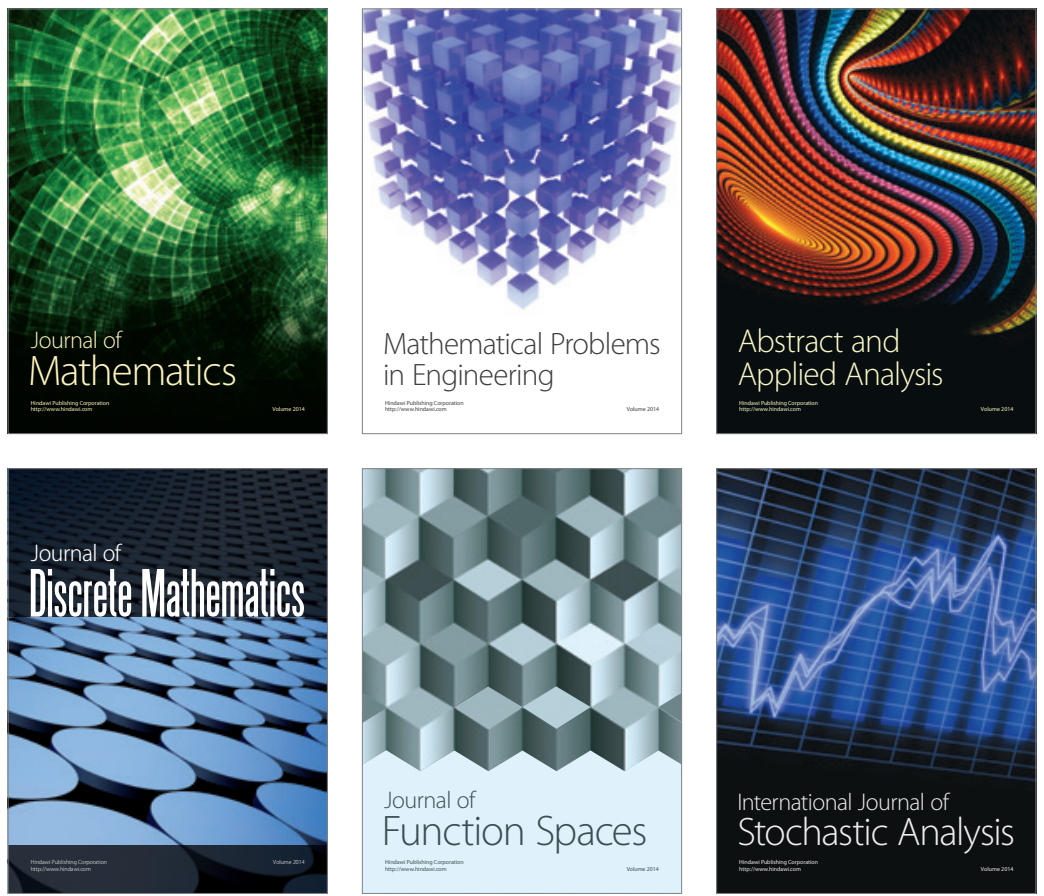

Journal of

Function Spaces

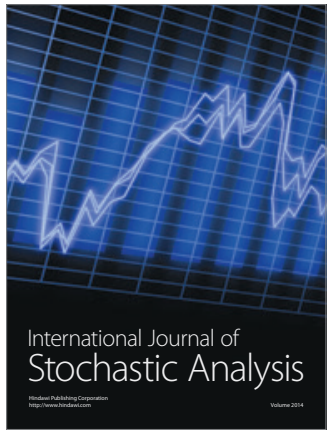

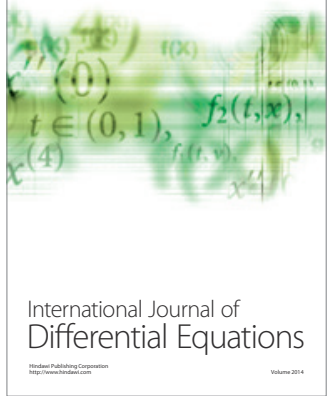
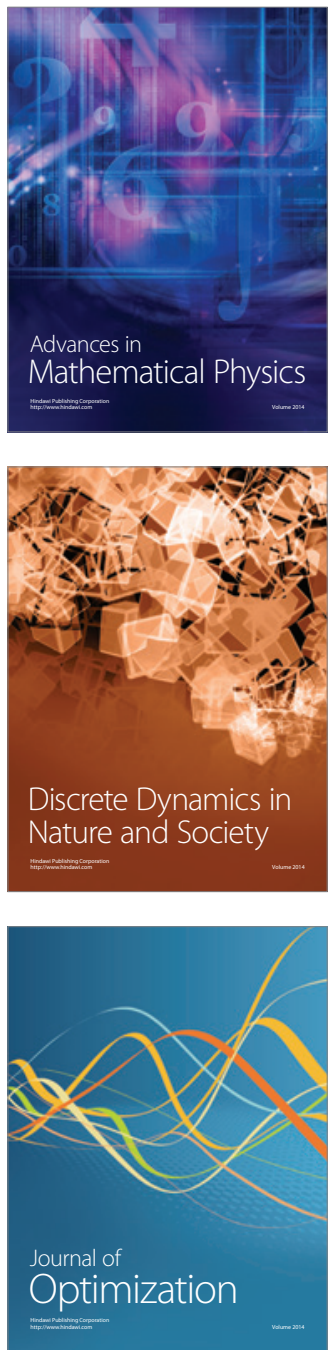